\title{
The Temporal Profile of OPG Expression and Regulatory Role on Ischemic Brain Injury in Rats After MCAo
}

\author{
Li Haijian ${ }^{\&}$ Sun Jincheng\& Wang Lin ${ }^{\&}$ Fu Yiting Zhao Chunzhen
}

Department of Pharmacology and Laboratory of Applied Pharmacology, College of Pharmacy, Weifang Medical University, Weifang 261053, China

${ }^{\&}$ Co-first author: These authors equally contributed to the work.

Corresponding author: Zhao Chun-zhen, Department of Pharmacology and Laboratory of Applied Pharmacology, College of Pharmacy, Weifang Medical University,Weifang 261053, China.E-mail: chunzh414@163.com

Article History Received 15 January 2021 Accepted 25 January 2021 Published 31 March 2021

Cite this Article Li Haijian, Sun Jincheng, Wang Lin, Fu Yiting, Zhao Chunzhen. The Temporal Profile of OPG Expression and Regulatory Role on Ischemic Brain Injury in Rats After MCAo [J].Medical Research, 2021.3(1):15, http://dx.doi.org/10.6913/MRHK.202103_3(1).0001

Copyright (2021 Creative Publishing Co., Limited.All rights reserved.Email:mrhk26640333@gmail.com.

\section{ABSTRACT}

Objective To investigate the temporal profile of osteoprotegerin (OPG) in middle cerebral artery occlusion (MCAo) rats and the serum level of RANKL and ALP in OPG deficient and wild type rats.

Methods Rats was anesthetized and subjected to MCAo by transient occlusion of middle cerebral artery occlusion. The serum level of OPG, RANKL and ALP in rats after MCAo was examined by ELISA assay. The protein expression of OPG in ischemic brain was determined by Western blot analysis.

Results The level of OPG in the rat serum was significantly increased from $6 \mathrm{~h}$ after MCAo and peaked at 1224 and 72-168 h. The protein expression of OPG was upregulated from $12 \mathrm{~h}$ after MCAo and peaked at $72 \mathrm{~h}$. The level of RANKL and ALP was significantly decreased in OPG deficient rats after MCAo.

Conclusion OPG/RANKL signaling is associated with brain injury after MCAo, indicating a potential therapeutic target for ischemic stroke.

Keywords cerebral ischemia; osteoprotegerin; receptor activator of nuclear factor- $\mathrm{\kappa B}$ ligand

\section{INTRODUCTION}

Stroke is one of the main cause of death and disability worldwide following cancer and cardiovascular diseases, rising with the increase of age ${ }^{[1]}$. The burden of ischemic stroke increased significantly and led to brain injury, edema, hemorrhage and neuronal death ${ }^{[2]}$. However, the pathogenesis is not fully understand. Hundreds of targeting chemicals were developed, but there are few effective drugs against stroke. 
Li Haijian, et.,al. The Temporal Profile of OPG Expression and Regulatory Role on Ischemic Brain Injury in Rats After MCAo Medical Research ISSN 2664-0333 eISSN 2664-0341 Volume 3 Issue $1 \quad$ http://dx.doi.org/10.6913/MRHK.202103_3(1).0001

Osteoprotegerin (OPG) is a soluble protein which bands to a receptor activator of nuclear factor- $\mathrm{\kappa B}$ ligand (RANKL) ${ }^{[3]}$. It is reported that elevated level of OPG in the serum was closely related to the severity and poor functional outcome of ischemic stroke ${ }^{[4-6]}$. OPG/RANKL signaling has been known to work on vascular calcification and immune system ${ }^{[7,8]}$. In the present study, we examined the temporal expression of OPG in ischemic brain and the regulation on RANKL and ALP in a transient middle cerebral artery occlusion model using $\mathrm{OPG}^{-/}$rats.

\section{MATERIALS AND METHODS}

\section{ANIMAL PROCEDURE}

All animal procedures were undertaken in accordance with the guidelines for animal care and welfare and were approved by the Ethical Committees of Weifang Medical University. All animals were kept at about 23 $\pm 2{ }^{\circ} \mathrm{C}$ and a $12 \mathrm{~h}$ light $/ 12 \mathrm{~h}$ dark cycle with free access to tap water and diet.

\section{TRANSIENT MIDDLE CEREBRAL ARTERY OCCLUSION (MCAO)}

Rats were randomly divided into sham operated group and MCAo group. Rats were anesthetized with intraperitoneal injection of chloral hydrate and subjected to MCAo as previously reported. In short, a silicon coated 4-0 monofilament nylon suture with a rounded tip was inserted from the right common carotid artery and gently advanced until it occlude the middle cerebral artery. The monofilament was gently removed after reperfusion. In sham-operated animals, all procedures were performed except occlusion of the middle cerebral artery. The animals received humane care and kept under $37^{\circ} \mathrm{C}$.

\section{ELISA}

The blood was obtained at the end of the experiment and centrifuged at $3000 \mathrm{rpm}$ for $15 \mathrm{~min}$ to collect serum. The level of osteoprotegerin (OPG), receptor activator for nuclear factor- $\kappa B$ ligand (RANKL) and alkaline phosphatase (ALP) in the plasma was determined using commercially available ELISA kit according to the manufacture's instructions.

\section{WESTERN BLOT ANALYSIS}

The brain tissues were homogenized in lysis buffer containing containing pepstatin 1, leupeptin 2, phenylmethyl -sulfonyl fluoride 1 . The lysates were centrifuged at $12000 \mathrm{rpm}$ for $30 \mathrm{~min}$ and the concentration was detected using coomassie brilliant blue staining method. $60 \mu \mathrm{g}$ of protein in each sample were separated using SPS-PAGE. The protein samples were transfered into polyvinylidene fluoride (PVDF) membranes for immunoblotting. The PVDF membranes were blocked using 7.5\% defatted milk. Then the primary antibody against OPG and GAPDH were used to incubate the membranes at $4{ }^{\circ} \mathrm{C}$ overnight. After incubated with antirabbit IRDye700DX ${ }^{\circledR}$-conjugated second antibody or anti-mouse IRDye800DX®-conjugated antibody (1:5000, Rockland, USA), the bands were detected by an Odyssey infrared imaging system. The intensity of the bands were quantitatively evaluated using Quantity One ${ }^{\circledR}$ analysis software.

\section{STATISTICAL ANALYSIS}

All data were presented as mean $\pm \mathrm{SD}$. The significance of differences among groups was assessed using one-way ANOVA followed by Bonferroni's post hoc test using the software Prism 5.0 ( GraphPad Software). 
$\mathrm{P}<0.05$ was considered statistically significant.

\section{RESULTS}

Temporal profile of OPG content and expression.

To investigate the regulatory role of OPG after ischemia, we examined the level of OPG in the rat serum after MCAo. As shown in Fig.1, the level of OPG in the serum was increased at $6 \mathrm{~h}$ after MCAo and peaked at 12-24 $\mathrm{h}$ and 72-168 $\mathrm{h}$ after MCAo. In rats, OPG protein expression in ischemic brain was increased and peaked at $12 \mathrm{~h}$ and $72 \mathrm{~h}$ after MCAo (Fig.2). These data indicate that OPG primarily acts in the acute stage of ischemic brain.

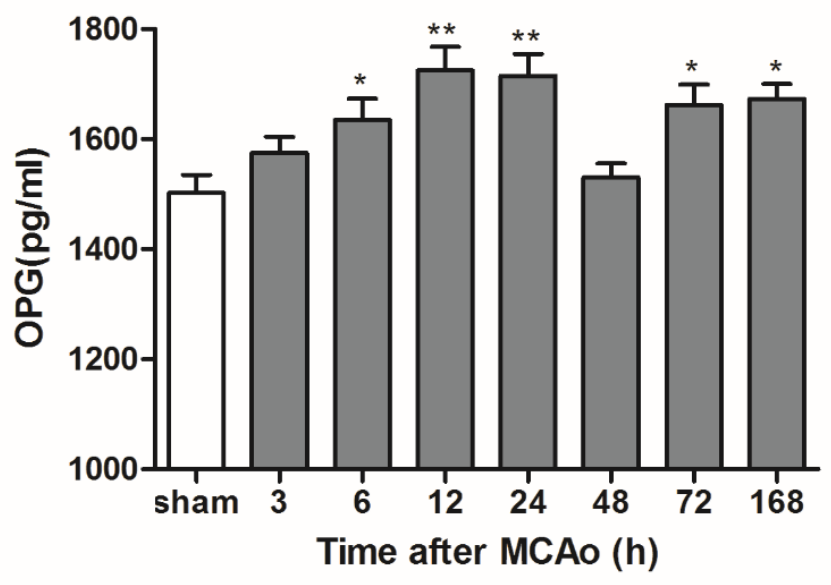

Fig.1 The level of OPG in rat serum after MCAo. The plasma OPG was examined using ELSIA assay at 3, 6,12 $24,48,72,168 \mathrm{~h}$ after MCAo in rats. $\mathrm{n}=4$ in each group. ${ }^{*} \mathrm{P}<0.05,{ }^{* *} \mathrm{P}<0.01$ vs sham-operated rats.

A

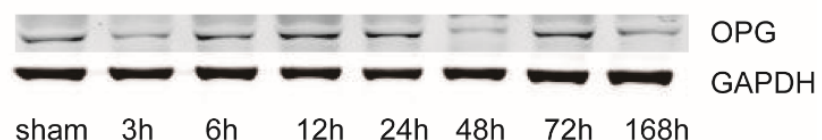

B

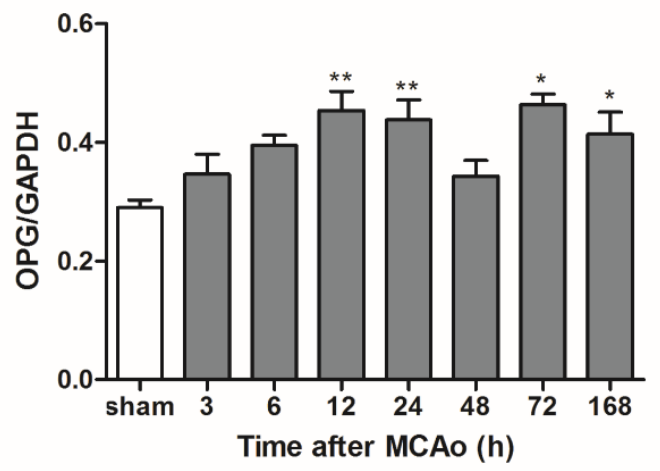

Fig.2 The protein expression of OPG in rats after MCAo. The expression of OPG protein was analyzed by

Western blot. $\mathrm{n}=3$ in each time point. ${ }^{*} P<0.05,{ }^{* *} P<0.01$ vs sham-operated rats.

\section{Changement of RANKL and ALP in the plasma of rats after MCAO.}

To elucidate the role of OPG/RANKL signaling, we examined the changes of RANKL and ALP in the plasma using ELISA detection assay. $24 \mathrm{~h}$ after MCAo, we examined the content of RANKL and ALP in the plasma in OPG-/- and WT rats. As shown in Fig.3A and 3B, the level of RANKL and ALP in the plasma was 
significantly increased $24 \mathrm{~h}$ after MCAo compared with OPG-/- sham-operated rats. The level of RANKL and ALP in OPG-/- rats was significantly decreased compared with that in WT rats after MCAo. These results indicated that the OPG/RANKL signaling played important roles in MCAo rats.

A

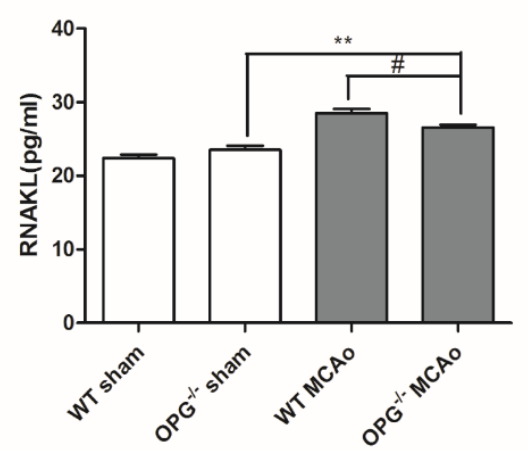

B

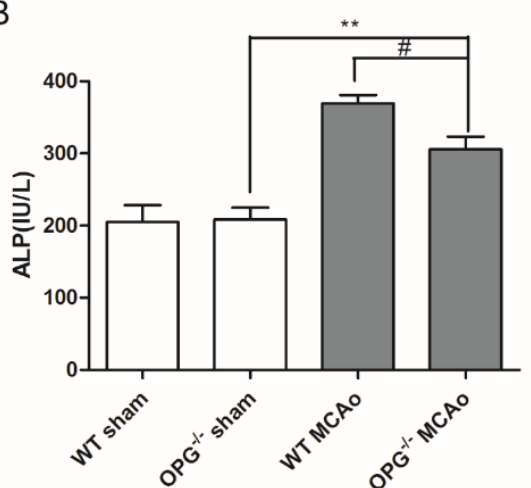

Fig.3 The content of RNAKL and ALP in the serum of rats. Serum RNAKL and ALP was examined using ELISA assay at $24 \mathrm{~h}$ after MCAo in $\mathrm{OPG}^{-/-}$rats. $\mathrm{n}=3$ in each time point. ${ }^{* *} P<0.05 \mathrm{vs} \mathrm{OPG}^{-/-}$sham operated rats, ${ }^{\#} P<$ 0.01 vs WT rats subjected to MCAo.

\section{DISCUSSION}

Stroke is the leading cause of mortality and disability in adults since millions of Chinese people died from ischemic stroke ${ }^{[9-11]}$. Therefore, it is important to develop more effective treatment of ischemic stroke. In the present study, we investigate the temporal expression of OPG in the plasma and ischemic brain in the middle cerebral artery occlusion for different points of reperfusion.

It has been reported that OPG/RANKL/RANK signaling was involved in brain ischemia ${ }^{[12,13]}$. Increased level of OPG in the serum has been observed in patients with ischemic stroke, which is related with the severity of stroke. The regulatory role of OPG in ischemic brain injury is not yet known. Here we demonstrated that the level of OPG protein in the infarct region was increased from 6 to $24 \mathrm{~h}$ after middle cerebral artery occlusion. The protein expression of OPG peaked again $72 \mathrm{~h}$ after MCAo. Moreover, in OPG deficient rat, the level of RANKL and ALP in the plasma was decreased significantly after ischemia. Further studies are necessary to clarify the mechanisms that how OPG deletion regulated the expression of RANKL.

The present study demonstrated that OPG/RANKL signaling could be associated with cerebral and other diseases in central nervous system. Further studies on the OPG/RANKL system in the brain might shed light on molecular mechanisms involved in brain injury and be a potential therapeutic target for ischemic stroke.

\section{ACKNOWLEDGEMENTS}

This work was supported by College Student Innovation and Entrepreneurship training program of Shandong province (S202010438046), Science and Technology Development Program of Traditional Chinese Medicine in Shandong Province (2017-207), Project of Shandong Province Higher Educational Science and Technology Program (J17KA256 and J18KA280).

\section{REFERENCES}

1. Wenzhi Wang, Bin Jiang, Haixin Sun, et al. Prevalence, Incidence, and Mortality of Stroke in 
Li Haijian, et.,al. The Temporal Profile of OPG Expression and Regulatory Role on Ischemic Brain Injury in Rats After MCAo Medical Research ISSN 2664-0333 eISSN 2664-0341 Volume 3 Issue $1 \quad$ http://dx.doi.org/10.6913/MRHK.202103_3(1).0001

China: Results from a Nationwide Population-Based Survey of 480687 Adults. Circulation, 2017; 135(8): 759-771.

2. Gang Zuo, Dongping Zhang, Rutao Mu, et al. Resolvin D2 protects against cerebral ischemia/reperfusion injury in rats. Mol Brain, 2018; 11(1): 9.

3. Munehisa Shimamura, Hironori Nakagami, Mariana K Osako, et al. OPG/RANKL/RANK axis is a critical inflammatory signaling system in ischemic brain in mice.Proc Natl Acad Sci U S A, 2014; 111(22): 8191-8196.

4. Xin Xiong, Duraid Hamied Naji, Binbin Wang, et al. Significant Association between OPG/TNFRSF11B Variant and Common Complex Ischemic Stroke. J Stroke Cerebrovasc Dis, 2018; 27(6): 1683-1691.

5. Federico Carbone, Nicolas Vuilleumier, Fabienne Burger, et al. Serum osteopontin levels are upregulated and predict disability after an ischaemic stroke. Eur J Clin Invest, 2015; 45(6): 579-586.

6. Hongqin Zhao, Yanyan Cao, Honghua Chen, et al. The association between OPG rs3102735 gene polymorphism, microembolic signal and stroke severity in acute ischemic stroke patients. Gene, 2017; 613: 25-29.

7. Emma Harper, Hannah Forde, Colin Davenport, et al. Vascular calcification in type-2 diabetes and cardiovascular disease: Integrative roles for OPG, RANKL and TRAIL.Vascul Pharmacol, 2016; 82: 3040.

8. Bowen Meng, Dongle Wu, Yangfan Cheng, et al. Interleukin-20 differentially regulates bone mesenchymal stem cell activities in RANKL-induced osteoclastogenesis through the OPG/RANKL/RANK axis and the NF-אB, MAPK and AKT signalling pathways. Scand J Immunol, 2020; 91(5): e12874.

9. Zhang Yang, Chen Weian, Huang Susu, et al. Protective effects of mangiferin on cerebral ischemia-reperfusion injury and its mechanisms. Eur J Pharmacol, 2016; 771: 145-151.

10. Gui-Cun Zhao, Yan-Ling Yuan, Feng-Rong Chai, et al. Effect of Melilotus officinalis extract on the apoptosis of brain tissues by altering cerebral thrombosis and inflammatory mediators in acute cerebral ischemia. Biomed Pharmacother, 2017; 89: 1346-1352.

11. Shamir Haji, Ryan Planchard, Adeel Zubair, et al. The clinical relevance of cerebral microbleeds in patients with cerebral ischemia and atrial fibrillation. J Neurol, 2016; 263(2): 238-244.

12. Reiko Hanada. The role of the RANKL/RANK/OPG system in the central nervous systems (CNS). J Bone Miner Metab, 2021; 39(1): 64-70.

13. Tae-Jin Song, Jinkwon Kim, Seung-Hee Yang, et al. Association of plasma osteoprotegerin levels with stroke severity and functional outcome in acute ischaemic stroke patients. Biomarkers, 2012; 17(8): 738-744. 